\title{
Numerical investigation of the effects of pedestrian barriers on aeroelastic stability of a proposed footbridge.
}

\author{
I J Taylor ${ }^{\mathrm{a},{ }^{*}}$, M Vezza $^{\mathrm{b}}$, I Salisbury $^{\mathrm{c}}$ \\ ${ }^{a}$ Dept of Mechanical Engineering, University of Strathclyde, Glasgow, G1 1XJ. \\ ${ }^{\mathrm{b}}$ Dept of Aerospace Engineering, University of Glasgow, Glasgow, G12 8QQ. \\ ${ }^{\mathrm{c}}$ Halcrow Group Ltd, City Park, 368 Alexandra Parade, Glasgow, G31 3AU
}

\begin{abstract}
:
A numerical investigation into the aerodynamic characteristics and aeroelastic stability of a proposed footbridge across a motorway in the north of England has been undertaken. The longer than usual span, along with the unusual nature of the pedestrian barriers, indicated that the deck configuration was likely to be beyond the reliable limits of the British design code BD 49/01. In particular, the investigation focussed on the susceptibility of the bridge due to flutter, and to assess if the design wind speeds could be met satisfactorily. The calculations were performed using the discrete vortex method, DIVEX, developed at the Universities of Glasgow and Strathclyde. DIVEX has been successfully validated on a wide range of problems, including the aeroelastic response of bridge deck sections. The proposed deck configuration, which incorporated a pedestrian barrier comprised of angled flat plates, was found to be unstable at low wind speeds with the plates having a strong turning effect on the flow at the leading edge of the deck. DIVEX was used to assess a number of alternative design options, investigating the stability with respect to flutter for each configuration. Reducing the number of flat plates and their angle to the deck lessened the effect of the barrier on the overall aerodynamic characteristics and increased the stability of the bridge to an acceptable level, with the critical flutter speed in excess of the specified design speed. Keywords : Flutter; Bridge; Vortex Method; CFD; Pedestrian Barrier
\end{abstract}

\section{INTRODUCTION}

Much research has been undertaken in the field of bridge aerodynamics, and techniques for predicting the onset of flutter instabilities have been established for many years (Scanlan and Tomko, 1971; Scanlan, 1978, 1997), with more advanced techniques still being proposed (Chen and Kareem, 2003; Matsumoto et al, 1996). Assessment of the structural integrity due to the unsteady wind loading is not only important for long span suspension bridges, but also for much shorter span footbridges as recent studies indicate (Flaga et al, 2002; Pirner and Fischer, 1998; Tanaka et al, 2002). In certain respects, footbridges could be more susceptible to aeroelastic effects as the deck section is much lighter and thinner relative to the overall span, and they also tend to be much more flexible than longer span bridges. The oscillation of the London Millennium

\footnotetext{
* Corresponding author. Tel.: +44 (0)141 548 3753; Fax: +44 (0)141 5525105

Email address : ian.taylor@strath.ac.uk
} 
Bridge in 2000 (Dallard et al, 2001) memorably demonstrated the flexibility of footbridges and susceptibility to oscillation, although in this case the forcing mechanism was not due to the unsteady wind loading.

Planners and architects are increasingly commissioning and designing unique structures that can be used to provide a distinct landmark for the city or town. Whilst these structures are dramatic and impressive, they pose an extra problem and challenge for the engineer because of their unique nature. In the early design stages for these bridges, the engineer will seek to assess structural loading, including aerodynamic loads, from the available design codes (for example British Standard BD 49/01). However, these codes use analysis methods and empirical correlations that are largely based on experience from previous designs or from experiments conducted on "generic" structures. Hence, design codes will provide limited information on novel "nonstandard" structures for which alternative analysis techniques such as numerical and computational methods are required.

Numerical methods are increasingly being used for aerodynamic analysis as part of the design procedure for numerous wind engineering structures. In particular, numerical techniques are considered to be particularly useful in the initial stages of the design, allowing designers to assess a range of options, to perform feasibility studies on novel configurations, or to provide useful aerodynamic and structural information on designs that lie beyond the scope of design codes.

A particular approach that is extremely well suited to assessing unsteady aerodynamic effects and structural integrity is the discrete vortex method, with numerous researchers now using this type of numerical procedure very successfully as a design tool for bridge deck design (Larsen \& Walther, 1997; MacKenzie et al, 2002; Taylor et al, 2002b; Vejrum et al, 2000).

Discrete vortex methods are based on the discretisation of the vorticity field rather than the velocity field, into a series of vortex particles, each of which is of finite core size and carrying a certain amount of circulation. The particles are tracked in time throughout the flow field that they collectively induce. As a result of this approach, the model does not require a calculation mesh and provides a very different method of analysis to more traditional grid based computational fluid dynamics methods. Full and comprehensive reviews of the discrete vortex method are given in Leonard (1980), Puckett (1993) and Sarpkaya (1989).

DIVEX is a two dimensional discrete vortex method that has been developed at the Universities of Glasgow and Strathclyde. To date, DIVEX has been used to analyse a range of bluff body flow fields (Taylor and Vezza. 1999a, b), and has also been extensively validated for a range of bridge deck analyses, ranging from predictions of static aerodynamics loads, flutter analysis and the study of flow control devices (Taylor and Vezza, 2001 and 2002a). The capability of DIVEX is now well recognised and the code has been used by Halcrow Group Ltd. during a number of recent design projects, a selection of which are summarised in Taylor et al (2002b). 
The results presented herein are based on a design study, commissioned by Halcrow Group Ltd., performed at the Universities of Glasgow and Strathclyde, on a proposed footbridge in the North of England. The study focused on assessing the structural integrity of the bridge with respect to flutter, as the proposed crossing was for a span longer than previously experienced by Halcrow for a bridge of this type, and also as the unusual pedestrian barriers were considered to be beyond the scope of the design code BD 49/01. This paper presents the results of the numerical analysis, which demonstrate the strong detrimental effect that these barriers have on the flutter instability, and the attempts to alleviate the problem by employing DIVEX to assess different design options relatively quickly. This analysis highlights the usefulness of DIVEX as part of a design process, and more generally demonstrates how numerical procedures can be used to provide information to assist engineers in the assessment of the structural integrity of new and unique designs.

\section{PROPOSED DESIGN}

The proposed bridge is a new pedestrian crossing across a widened highway in the north of England. The design of the bridge is based on previous Halcrow designs for pedestrian crossings, to build upon and utilise previous knowledge and experience, but also to provide an element of "corporate identity" for the bridge. However, two uncertainties in the proposed design led to Halcrow commissioning a numerical investigation into the aerodynamic characteristics and aeroelastic stability of the bridge. Firstly, as the bridge will be crossing a widened highway, it has a span significantly longer than previous similar designs. Hence, the potentially lower structural stiffness of this longer span raised a few concerns over its structural integrity, necessitating the requirement to assess the aeroelastic stability of the proposed bridge with respect to both flutter and vortex induced vibration. Secondly, the existing bridges of this type designed and constructed by Halcrow were not only pedestrian crossings but also part of a bridle path where there would be a significant number of crossings by riders on horseback. For this reason, the barriers along the edges of the deck comprised a series of angled flat plates, designed specifically to block the horse's view of vehicular movement underneath the bridge, thus preventing the horse being startled whilst crossing the bridge. Although this new footbridge is not part of a bridle path, it was decided that the angled flat plates be retained as part of the initial design, to maintain consistency with previous designs. However, the design of these "angled vanes" meant that the configuration of the bridge deck was outside the scope of the design code BD $49 / 01$.

A simplified schematic diagram of the proposed initial design is illustrated in Figure 1 with some structural properties of the bridge indicated in Table 1. Also included in Figure 1 are relevant dimensions of the barriers and deck, which are used to ascertain the applicability of BD 49/01. The design guidelines place geometric constraints on the applicability of the design code, Eq. (1). These indicate that the solidity ratio of the barrier, $\phi$, should be less than 0.5 and that the product of the barrier height, $h$, (Figure 1) 
and the solidity ratio, $\phi$, should be less than $35 \%$ of the depth of the bridge deck, $d_{4}$ (from Section 2.3 - BD 49/01).

$$
\text { Geometric Constraints }\left\{\begin{array}{c}
\phi<0.5 \\
h \phi<0.35 d_{4}
\end{array}\right.
$$

The nature of the barriers on the bridge deck, and their orientation to the oncoming flow, mean that it is difficult to select an appropriate value of the solidity ratio, $\phi$. However, considering the overall frontal area of the barrier, it is reasonably likely (Figure 1) that $\phi>0.5$, contradictory to the geometric constraints, Eq. (1). Also, from the dimensions of the bridge deck (Table 1 ) for the product $h \phi$ to be less that $35 \%$ of the deck depth, then the solidity would need to be less than 0.09 .

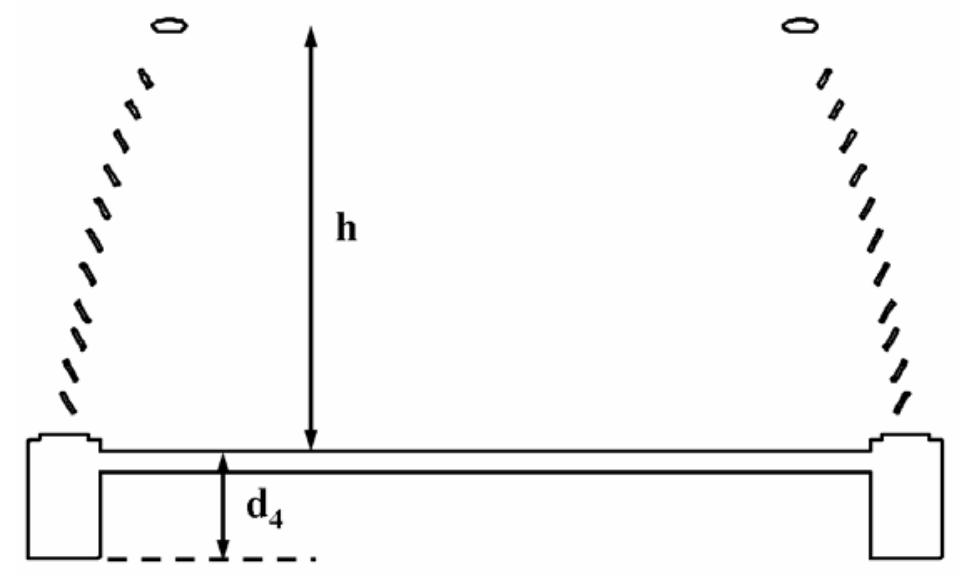

Figure 1. Schematic diagram of initial deck design.

Table 1.

Structural properties of proposed bridge design.

\begin{tabular}{ll}
\hline Structural Properties & Proposed Footbridge \\
\hline Bridge Deck Width, $B$ & $3.8 \mathrm{~m}$ \\
Mass per unit span, $m$ & $1427.6 \mathrm{~kg} / \mathrm{m}$ \\
$\begin{array}{l}\text { Moment of inertia per unit span, } I_{\alpha} \\
\quad\left(2^{\text {nd }} \text { moment of mass) }\right.\end{array}$ & $1172 \mathrm{kgm}^{2} / \mathrm{m}$ \\
$1^{\text {st }}$ bending (vertical DOF) frequency, $f_{h}$ & $1.42 \mathrm{~Hz}$ \\
$1^{\text {st }}$ torsional frequency, $f_{\alpha}$ & $3.06 \mathrm{~Hz}$ \\
Critical damping ratio, $\zeta_{h}, \zeta_{\alpha}$ & $0.5 \%$ \\
$\quad \quad$ vertical and torsional DOF) & $1.225 \mathrm{~kg} / \mathrm{m}^{3}$ \\
Density of Air, $\rho$ &
\end{tabular}

Another factor with the distinctive barrier design is that they are likely to have a significant impact on the overall aerodynamic characteristics of the bridge. The angled plates that form the pedestrian barrier will have a strong turning effect on the oncoming 
flow, which may have a significant effect on the flow field above the upper surface of the deck. Hence, the main objectives of the numerical study were to assess both the structural integrity of the bridge deck and the impact of the barrier configuration on the aerodynamic characteristics of the proposed bridge.

\section{NUMERICAL MODELLING}

\subsection{Discrete Vortex Method}

The numerical technique used in the analysis was DIVEX, a two-dimensional discrete vortex method developed at the Universities of Glasgow and Strathclyde. The model is an unsteady aerodynamic solver for incompressible and viscous flow fields. In this approach, the vorticity field is discretised into a series of vortex particles, rather than the more traditional approach utilised in grid based CFD where the velocity field is discretised. The detailed numerical implementation of the model is given in Lin, 1997 and Taylor and Vezza, 1999a. DIVEX can be used purely as an aerodynamic tool or in full aeroelastic mode where the response of structures to the unsteady flow can be assessed. In the former case, both static and moving bodies can be modelled as demonstrated in previous analyses, ranging from square and rectangular cylinders to suspension bridge deck sections and flow control devices (Taylor and Vezza, 1999a, 2001, 2002a).

\subsubsection{Mathematical formulation.}

Two dimensional incompressible viscous flow is governed by the vorticity-stream function form of the continuity and Navier-Stokes equations as given in Eq. (2) and Eq. (3) :

$$
\text { Continuity equation : }
$$

$$
\nabla^{2} \Psi=-\omega
$$

Vorticity transport equation :

$$
\frac{\partial \vec{\omega}}{\partial t}+(\overrightarrow{\mathbf{U}} . \nabla) \vec{\omega}=v \nabla^{2} \vec{\omega}
$$

where the vorticity, $\vec{\omega}$, is defined as the curl of the velocity, Eq. (4), and $\overrightarrow{\boldsymbol{\Psi}}$ is a vector potential defined by Eq. (5)

$$
\begin{aligned}
& \vec{\omega}=\nabla \times \overrightarrow{\mathbf{U}} \quad \text { with } \quad \vec{\omega}=\Psi \overrightarrow{\mathbf{k}} \\
& \overrightarrow{\mathbf{U}}=\nabla \times \overrightarrow{\boldsymbol{\Psi}}, \quad \nabla . \overrightarrow{\boldsymbol{\Psi}}=0, \quad \text { with } \quad \overrightarrow{\boldsymbol{\Psi}}=\Psi \overrightarrow{\mathbf{k}}
\end{aligned}
$$

The vorticity transport equation, Eq. (3), defines the motion of vorticity in the flow due to convection and diffusion. By using Green's Theorem, the velocity field can be calculated using the Biot-Savart law, which expresses the velocity in terms of the vorticity field. For a point $p$ outside the solid region, the velocity is given by :

$$
\overrightarrow{\mathbf{U}}_{p}=\overrightarrow{\mathbf{U}}_{\infty}+\frac{1}{2 \pi} \int_{F} \omega \frac{\overrightarrow{\mathbf{k}} \times\left(\overrightarrow{\mathbf{r}}_{p}-\overrightarrow{\mathbf{r}}\right)}{\left\|\overrightarrow{\mathbf{r}}_{p}-\overrightarrow{\mathbf{r}}\right\|^{2}} d F+\int_{B_{i}} 2 \Omega_{i} \frac{\overrightarrow{\mathbf{k}} \times\left(\overrightarrow{\mathbf{r}}_{p}-\overrightarrow{\mathbf{r}}\right)}{\left\|\overrightarrow{\mathbf{r}}_{p}-\overrightarrow{\mathbf{r}}\right\|^{2}} d B_{i}
$$


The pressure distribution on the body surface can be evaluated by integrating the pressure gradient along the body contour which at node $j$ on the body surface, is given by Eq. (7) (Lin, 1997).

$$
\frac{1}{\rho} \frac{\partial P}{\partial s}=-\overrightarrow{\mathbf{s}} \cdot \frac{D \overrightarrow{\mathbf{U}}_{c}}{D t}-\overrightarrow{\mathbf{n}} \cdot\left(\overrightarrow{\mathbf{r}}-\overrightarrow{\mathbf{r}}_{c}\right) \frac{D \Omega}{D t}+\overrightarrow{\mathbf{s}} \cdot\left(\overrightarrow{\mathbf{r}}-\overrightarrow{\mathbf{r}}_{c}\right) \Omega^{2}+v \frac{\partial \omega}{\partial n}
$$

The first three terms on the RHS in Eq. (7) are due to the body motion and represent the surface tangential components of the body reference point acceleration, the rotational acceleration and the centripetal acceleration. The final term is the negative rate of vorticity creation at the body surface and is calculated from the vorticity distribution created in the control zone between time $t-\Delta t$ and $t$. The resulting pressure distribution is integrated around the body surface to calculate the aerodynamic forces on the body.

\subsection{Numerical analysis}

The numerical analysis for this investigation consisted of a series of simulations, both static and dynamic, for each bridge deck option. The static analysis of the bridge deck was undertaken at a range of angles of incidence, between $+5^{\circ}$ and $-5^{\circ}$, with the mean lift and drag coefficients normalised using the deck width, $B$, and the moment coefficient normalised using the deck width squared. Positive lift is in the upwards direction and positive moment (and angle) is in the clockwise direction (positive moment tends to twist leading edge of the deck upwards) (Figure 2).

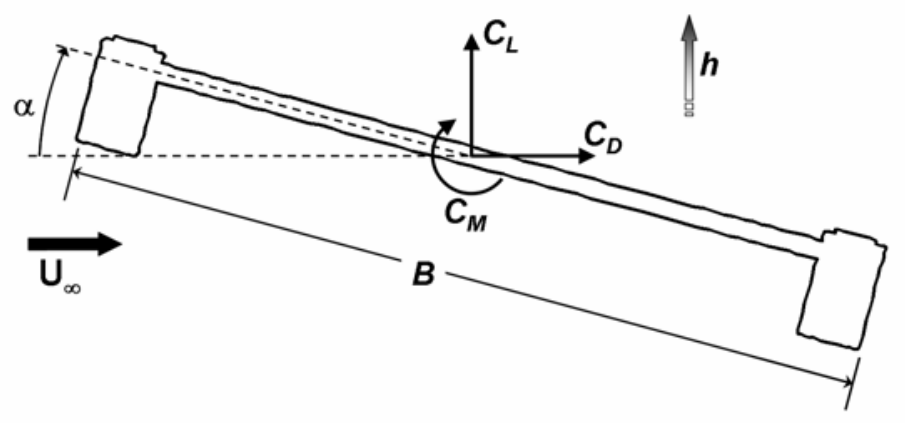

Figure 2. Frame of reference and indication of positive directions for bridge deck analysis.

Root mean square (RMS) quantities are also calculated to give a measure of the fluctuation of various parameters about the mean value throughout the analysis. In each case, the RMS fluctuation is calculated using Eq. (8), where $C$ is the parameter for which the RMS value is being determined (e.g. pressure coefficient, velocity, aerodynamic force etc. ), and $N$ is the number of samples (timesteps) from the time history that are used to calculate the mean and RMS quantities.

$$
C_{R M S}=\sqrt{\frac{\sum_{i=1}^{N}\left(C_{i}-\bar{C}\right)^{2}}{N}}
$$


In the dynamic analysis, determination of the flutter instability was investigated through a forced oscillation technique, using the traditional flutter derivatives (Simui and Scanlan, 1996), where the lift and moment coefficients are defined as :

$$
\begin{gathered}
L=\frac{1}{2} \rho U_{\infty}^{2}(2 B)\left[k H_{1}^{*}(k) \frac{\dot{h}}{U_{\infty}}+k H_{2}^{*}(k) \frac{B \dot{\alpha}}{U_{\infty}}+k^{2} H_{3}^{*}(k) \alpha+k^{2} H_{4}^{*}(k) \frac{h}{B}\right] \\
M=\frac{1}{2} \rho U_{\infty}^{2}\left(2 B^{2}\right)\left[k A_{1}^{*}(k) \frac{\dot{h}}{U_{\infty}}+k A_{2}^{*}(k) \frac{B \dot{\alpha}}{U_{\infty}}+k^{2} A_{3}^{*}(k) \alpha+k^{2} A_{4}^{*}(k) \frac{h}{B}\right]
\end{gathered}
$$

and $k$ is the non-dimensional reduced frequency

$$
k=\frac{\omega B}{U_{\infty}}=\frac{2 \pi f B}{U_{\infty}}
$$

During this analysis, the bridge deck motion, in either the vertical or torsional DOF, was modelled using a series of forced sinusoidal oscillations with an amplitude of 5\% of the bridge deck width, $B$, in the vertical degree of freedom, and $4^{\circ}$ in the torsional degree of freedom.

\subsection{Design modifications}

Four different deck designs were analysed during the study, beginning with the basic deck section with no pedestrian barriers to provide a datum case for comparison. Also initially investigated was the proposed design (Option 1) with the pedestrian barrier comprising angled plates extending up to the height of the pedestrian hand rail (Figure 3 ). Based on the results of the static and dynamic analyses of these two cases, the barrier was redesigned in an attempt to mitigate the strong effect of the angled flat plates. Each redesign was analysed numerically using DIVEX to assess if the modifications had had a positive effect on the structural integrity of the bridge deck. Two re-designs, Options 2 and 3, which are discussed in more detail in the following sections, were considered that incorporated modifications to both the height and incidence of the flat plates in the pedestrian barrier (Figure 3). Each of the design options described above are also summarised in Table 2.

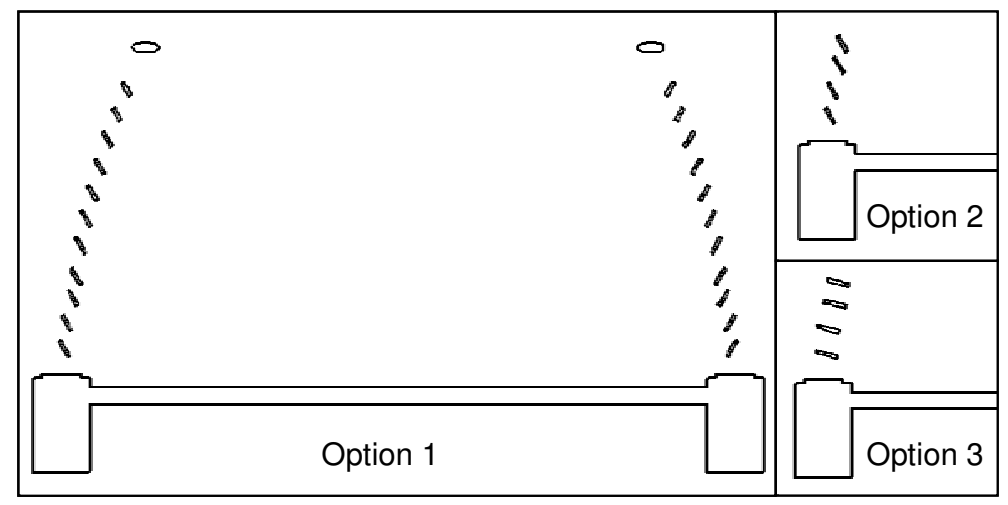

Figure 3. Schematic diagram of three barrier options. 
Table 2.

Summary of the design modifications analysed using DIVEX.

\begin{tabular}{ll}
\hline Deck Option & Description \\
\hline Basic Deck Section & $\begin{array}{l}\text { No pedestrian barriers - Deck section comprised of } 2 \text { rectangular box } \\
\text { section tubes, with concrete slab footway. } \\
\text { Steeply angled flat plates on each side of deck up to the height of the } \\
\text { pedestrian hand rail. }\end{array}$ \\
Parapet Option 2 & 4 steep angled flat plates on each side of deck. \\
Parapet Option 3 & 4 shallow angled flat plates on each side of deck. \\
\hline
\end{tabular}

\section{STATIC ANALYSIS}

\subsection{Aerodynamic Force Coefficients}

Unsteady time histories of the aerodynamic force coefficients are obtained from the DIVEX calculations on the static bridge deck. The calculations are for an impulsively started flow and hence the initial portion of the time history, where the flow field is developing, is omitted from the calculation of the mean aerodynamic force coefficients. The mean aerodynamic loads for the basic deck section and each barrier option, at $0^{\circ}$ incidence, along with the lift and moment slopes are shown in Table 3. The variations of the aerodynamic force coefficients with incidence are illustrated in Figure 4.

The presence of the angled plates has a significant effect on the static loads, noticeably reducing the mean lift coefficient and increasing moment coefficient at $0^{\circ}$. In effect, the flat plate barriers act as guide vanes, redirecting the oncoming flow downwards towards the top surface of the deck, and thus have a significant influence on the aerodynamic loads, particularly as $\alpha$ is varied. The plates at the windward side of the deck experience an upwards reaction force as the flow is directed downwards toward the upper deck surface. Similarly, the leeward plates experience a downward force as they direct the flow upwards (Figure 5), thus giving a significant increase in moment about the deck centre (Figure 4).

On the basic deck section without any barriers, the bluff leading edge of the deck directs the freestream away from the horizontal, causing a region of separated flow on the upper surface. This region of separated flow produces high suction pressures on the upper deck surface giving rise to the generally positive lift coefficient for the basic deck section (Figure 4). The barriers tend to direct the flow downwards at the bridge leading edge, suppressing the separation region, so that the suction pressures and hence the lift coefficient are reduced. This effect is clear in the variation of the lift coefficient with angle of incidence, $\alpha$, where all cases with barriers show a reduction in lift compared to the basic deck. As expected, the effect is most pronounced in Option 1, where the barriers are full height. 


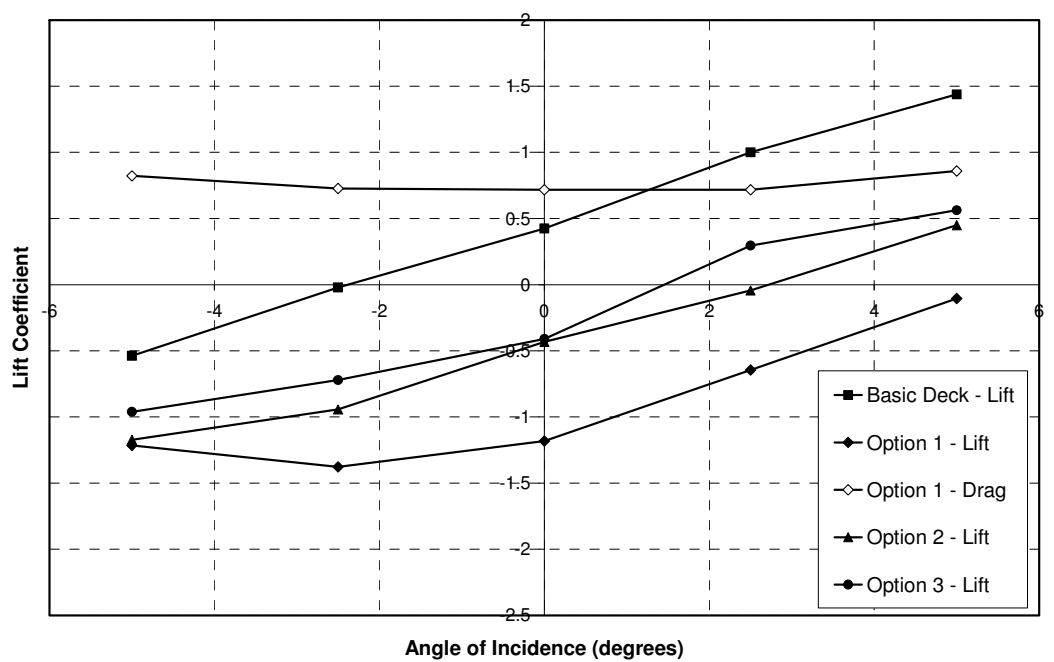

a) Lift Coefficient

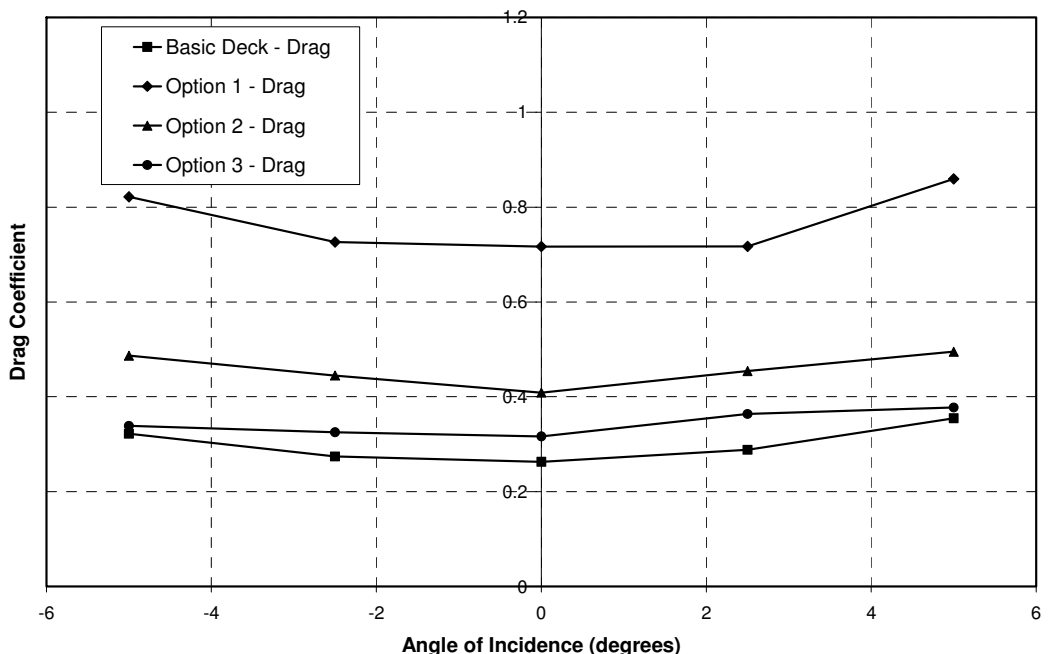

b) Drag Coefficient

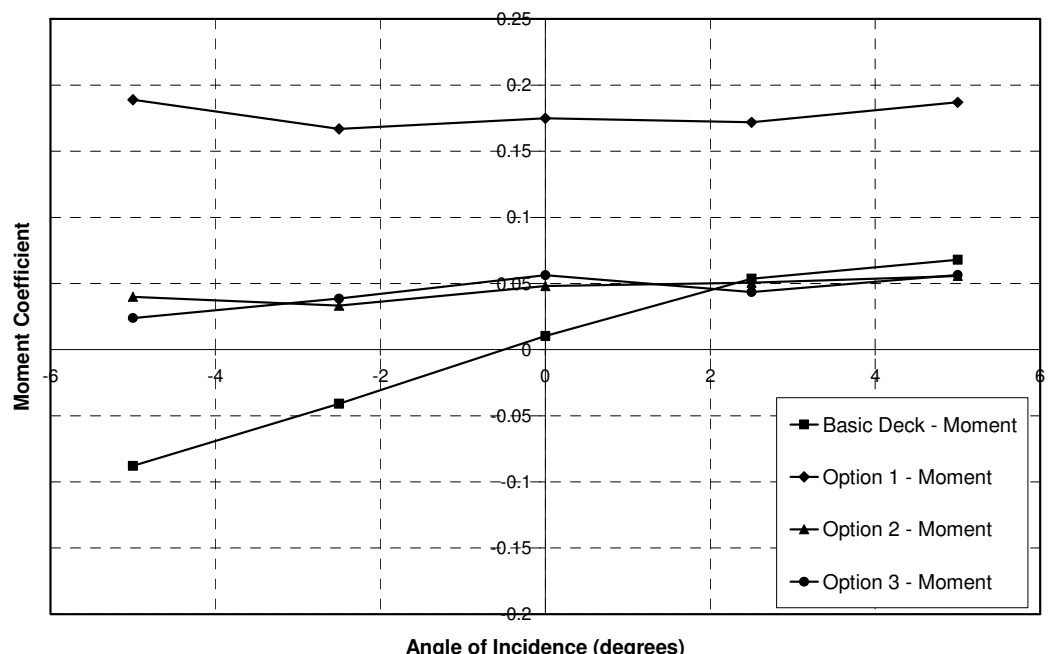

c) Moment Coefficient

Figure 4. Aerodynamic force coefficient vs. angle of incidence for basic deck and barrier options. 


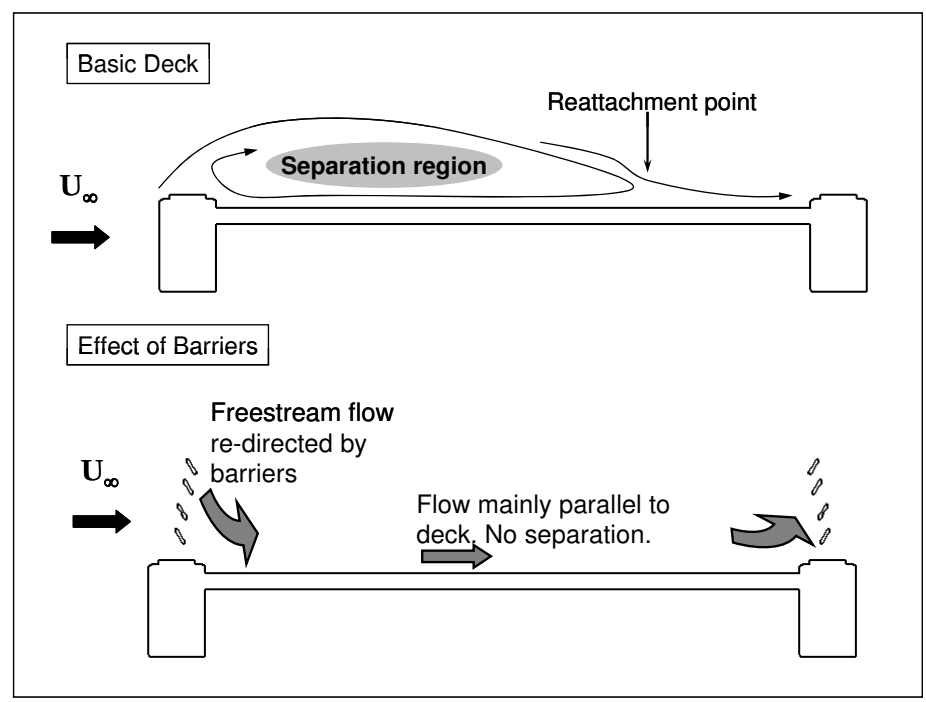

Figure 5. Schematic diagram of a typical flow field around the basic deck section and for the deck with barriers included.

Table 3.

Summary of Aerodynamic Loads on each Bridge Deck Option at $0^{\circ}$ Incidence.

\begin{tabular}{lccccc}
\hline Configuration & Lift Coef. & Drag Coef. & $\begin{array}{c}\text { Moment } \\
\text { Coef. }\end{array}$ & $\begin{array}{c}\text { Lift slope } \\
\left(\mathrm{rad}^{-1}\right) \\
d C_{L} /\left.d \alpha\right|_{\alpha=0}\end{array}$ & $\begin{array}{c}\text { Moment } \\
\text { slope } \\
\left(\mathrm{rad}^{-1}\right) \\
d C_{M} /\left.d \alpha\right|_{\alpha=0}\end{array}$ \\
\hline $\begin{array}{l}\text { Basic deck section } \\
\begin{array}{l}\text { Option } 1: \text { Full } \\
\text { height flat plates. }\end{array}\end{array}$ & 0.425 & 0.263 & 0.0103 & 11.70 & 1.083 \\
$\begin{array}{l}\text { Option 2 }: 4 \text { steep } \\
\text { flat plates. }\end{array}$ & -1.183 & 0.717 & 0.175 & 8.41 & 0.0573 \\
$\begin{array}{l}\text { Option 3 : } \\
\text { shallow flat plates. }\end{array}$ & -0.410 & 0.433 & 0.0481 & 10.32 & 0.198 \\
\hline
\end{tabular}

In general, the drag coefficient follows the expected trends, increasing due to the inclusion of barriers (maximum for full height barriers) and with angles of incidence away from $0^{\circ}$ (Figure 4 ).

Another important feature of the static characteristics for the cases with barriers, is the reduction of the moment slope by an order of magnitude (Table 3). With the flat plates in the barrier effectively acting as guide vanes, directing the flow down onto the upper surface of the deck at the windward side, at angles of incidence away from $0^{\circ}$, the turning effect of the flat plate barriers ensure that the flow along the upper surface of the deck remains relatively unchanged. Hence, the variation in upper surface pressures with angle of incidence is significantly reduced compared to the basic deck. For this reason, the proportionate increase in moment with incidence does not occur when the barriers are included, and thus the moment slope is greatly reduced. It should be noted that a low 
value of moment slope is an indication of potential susceptibility to single degree of freedom torsional instability.

This effect can also be seen by assessing the RMS lift and moment coefficients for each deck option (Figure 6). In general, at angles of incidence away from $0^{\circ}$, the RMS coefficients would be expected to increase due to the increased regions of separated flow (both above and below the deck) at higher angles, and the increased fluctuations in the flow that would arise as a result of the increased separation. This can be seen for the basic deck section, but for all the other cases, the RMS fluctuations reduce at incidence, indicative of the flow over the upper surface, and hence separation, being controlled by the flat plate barriers.
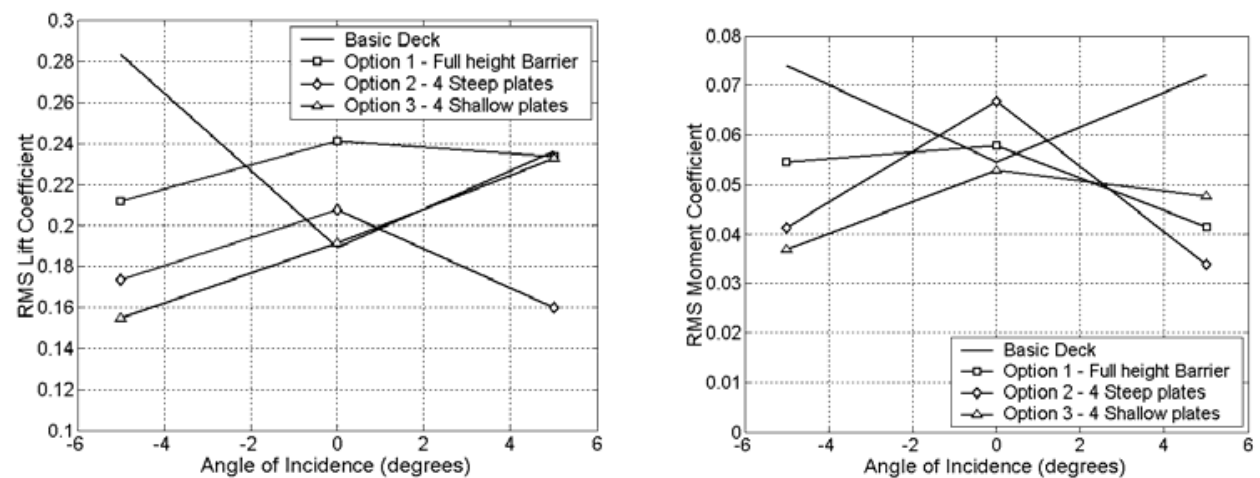

Figure 6. RMS lift and moment coefs. vs. angle of incidence for basic deck and barrier options.

\subsection{Mean and RMS velocity Fluctuations}

Mean velocity vectors for the basic deck and 3 options are illustrated in Figure 7, with mean and RMS velocity fluctuations shown in Figures 8 and 9 respectively. In each case, the mean velocity is normalised with respect to the freestream flow, which approaches from the left, and all dimensions are normalised with respect to the deck width, $B$.

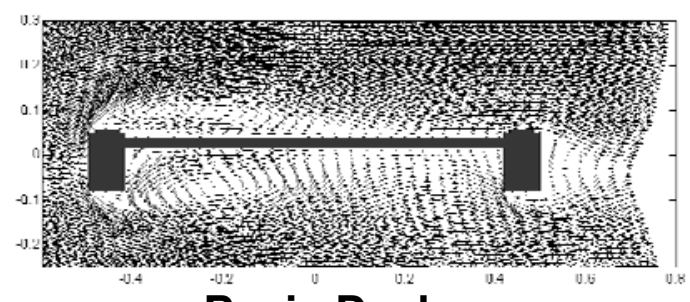

Basic Deck

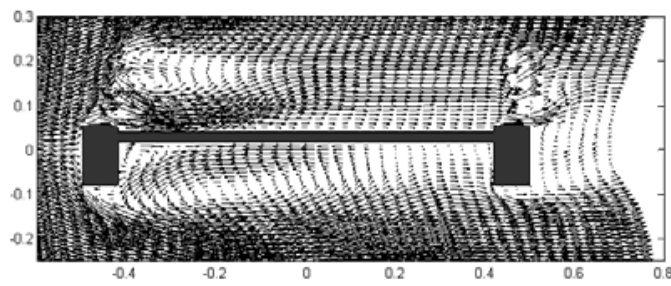

Option 2

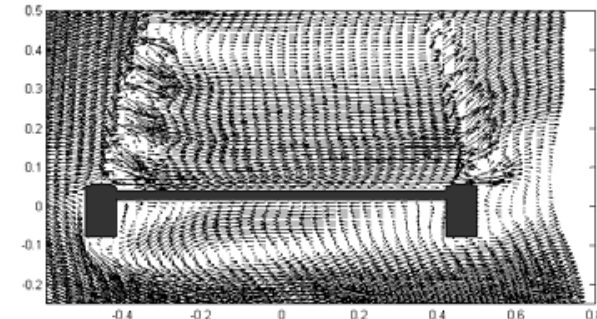

Option 1

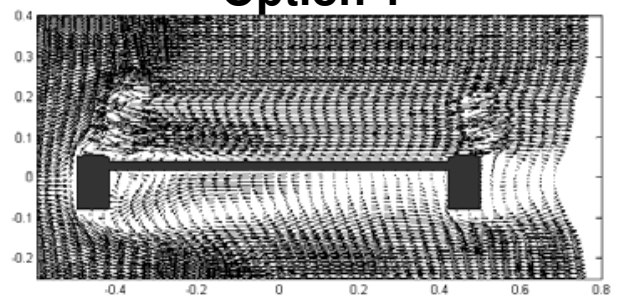

Option 3

Figure 7. Mean velocity vectors for all deck options. 
The flow field around the basic deck section follows the expected pattern, that is the flow stagnates on the front face of the deck, and a separated region develops over the upper surface, reattaching at around $60-70 \%$ of the deck width. Also, large velocity fluctuations are shown in the region of separated flow. When the full height barriers were included in Option 1, the strong influence of the flat plates on the flow over the upper surface of the deck is clear. Notably, the velocity vector plot clearly demonstrates the effect of the barrier on the windward side, turning the flow strongly down onto the deck surface, completely suppressing the region of separated flow over the upper surface. Similarly, the flat plates at the leeward edge of the deck deflect the flow strongly upwards. With the flat plates being at the extreme edges of the deck section, the flow deflection has a particularly strong effect on the aerodynamic loads over a range of incidence, and is the main reason for the low values of moment slope when the barriers are included (Table 3). Also, the suppression of the separated flow has significantly reduced the RMS fluctuations along the upper surface of the deck.

Similar effects are noticeable in deck options 2 and 3, with flow deviation onto the deck surface by the windward barrier, suppressing the region of separated flow, and greatly reducing the fluctuating velocity component. A particular feature of Option 2 is that the steep angled plates seem to generate much greater acceleration of the flow down onto the upper surface of the deck, and also produce a large fluctuating component behind the barriers on the windward side. These effects are mitigated to a large extent by the reduction in the angle of the flat plates in deck option 3.

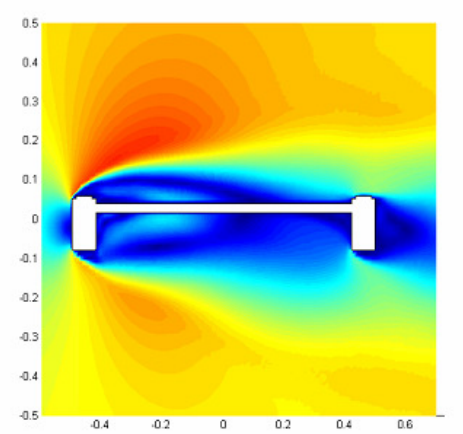

Basic Deck

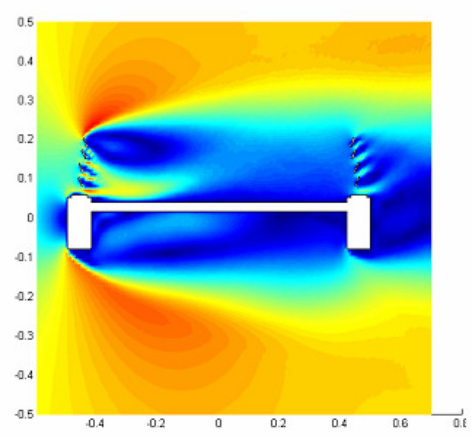

Option 2

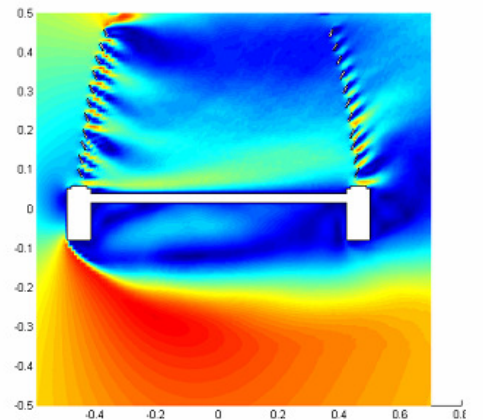

Option 1

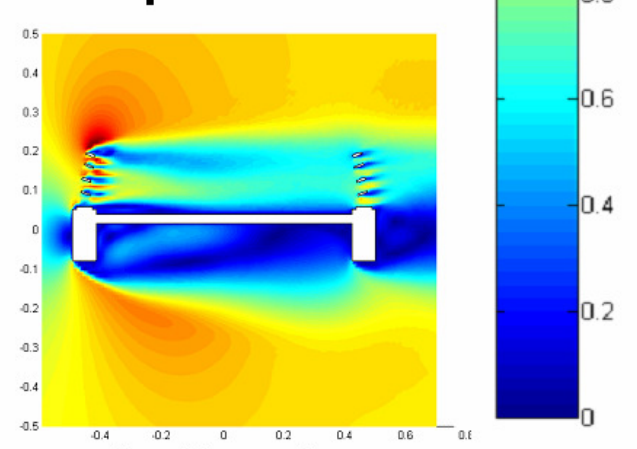

Option 3

Figure 8. Contours of mean velocity for all deck options. 


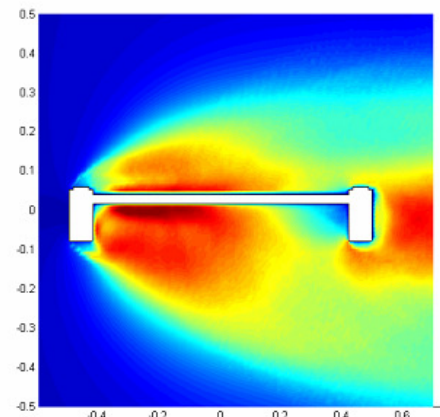

Basic Deck

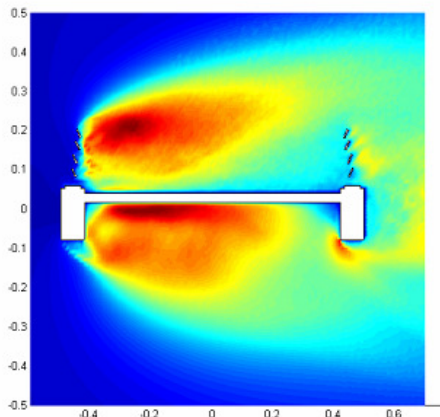

Option 2

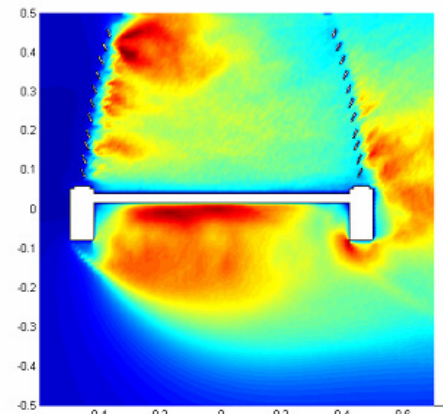

Option 1

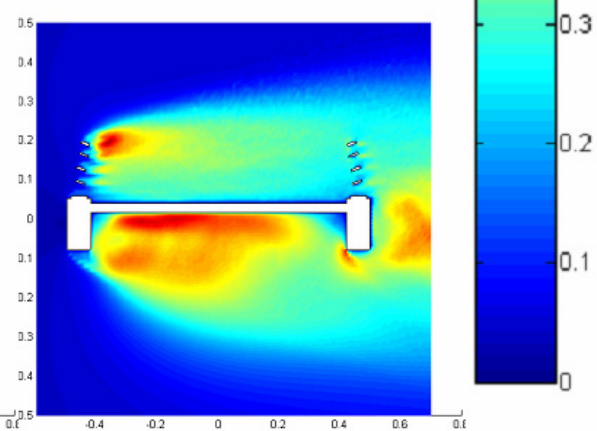

Option 3

Figure 9. Contours of RMS velocity fluctuations for all deck options.

\subsection{Mean and Fluctuating Pressures for Static Analysis}

The mean pressure coefficients and RMS fluctuating pressures for each deck option are shown in Figure 10 to Figure 12, for three angles of incidence. As the flow field on the underside of the deck is largely unchanged, for clarity, only the data along the upper surface of the deck is illustrated. The pressure distributions are plotted against the percentage non-dimensional horizontal distance along the deck, with the deck illustrated along with each pressure plot for reference ${ }^{1}$.

For the basic deck section at $0^{\circ}$ incidence (Figure 10), the separated region of flow is clear, with a region of large suction pressures up to around 60-70\% of the deck width, consistent with the results illustrated in the contours of mean velocity. Downstream of the reattachment point, the pressure coefficient is approximately constant at low suction pressures. The separated region is also clear in the RMS pressure coefficient, with large fluctuations over most of the upper surface of the deck. In each of the cases where the barriers are included, the large suction pressures are significantly reduced, and the mean pressure tends to show much less variation over the whole of the upper surface. Also, the pressure fluctuations are significantly reduced by the inclusion of the barriers, again

\footnotetext{
${ }^{1}$ The deck is non-dimensionalised with respect to the deck width, $B$, with the "origin" taken to be at the centre of the deck, the leading edge at -0.5 and the leeward edge at +0.5 . In Figure 23 to Figure 25 , the horizontal distance is presented as a percentage of deck width, from $-50 \%$ to $+50 \%$.
} 


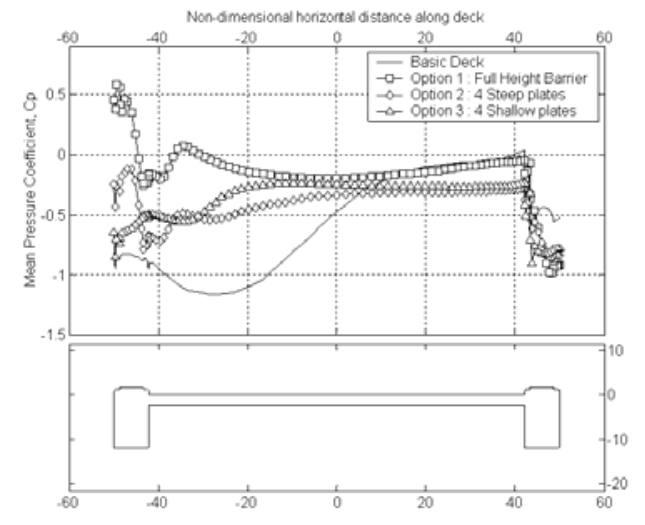

a)

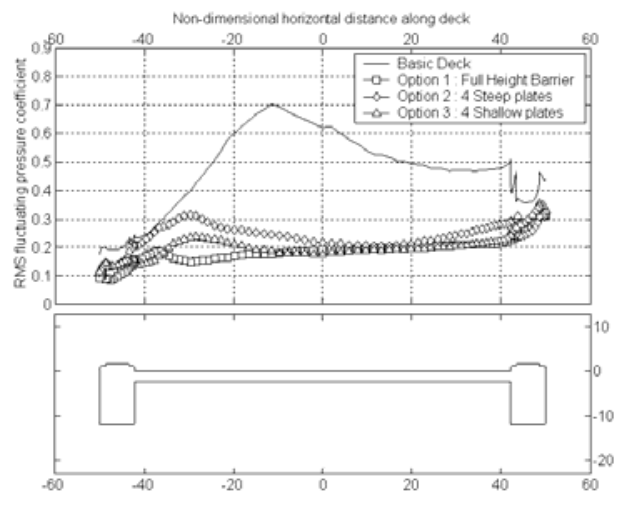

b)

Figure 10. Mean pressure coefficient and RMS fluctuating pressure coefficient on upper surface of deck at $0^{\circ}$ incidence : a) Mean pressure coefficient; b) RMS fluctuating pressure coefficient.

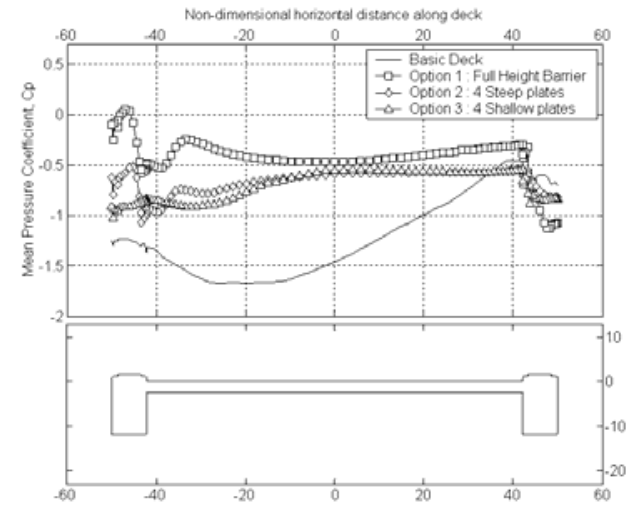

a)

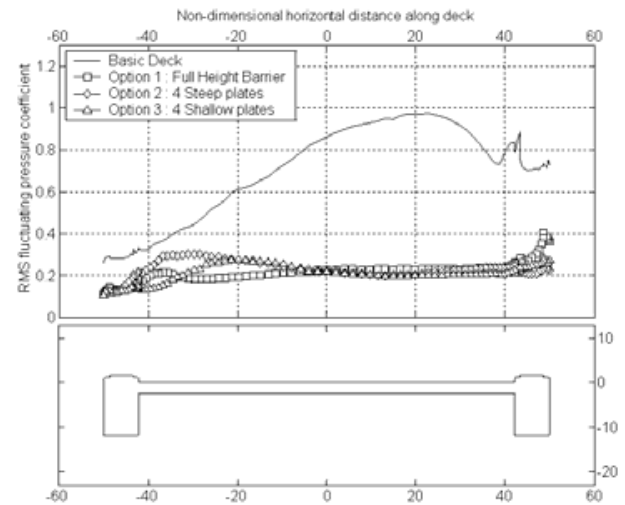

b)

Figure 11. Mean pressure coefficient and RMS fluctuating pressure coefficient on upper surface of deck at $+5^{\circ}$ incidence : a) Mean pressure coefficient; b) RMS fluctuating pressure coefficient.

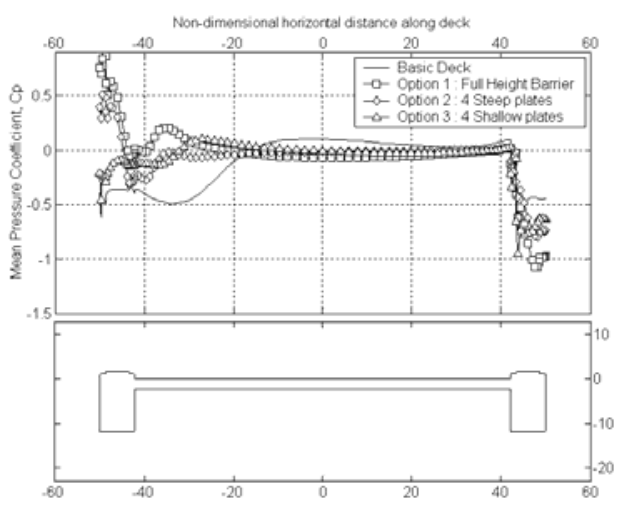

a)

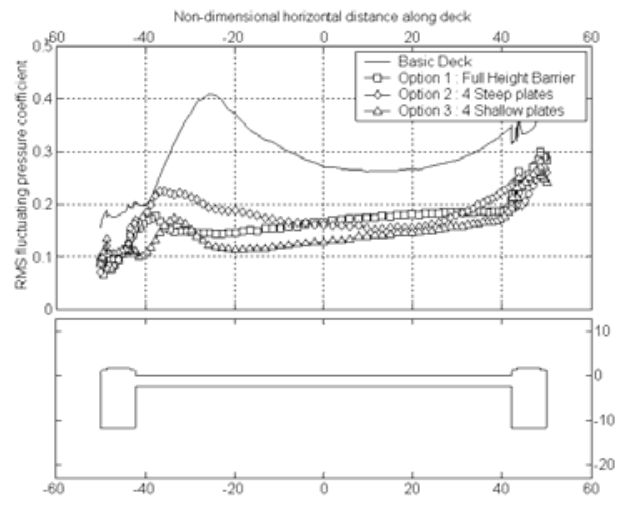

b)

Figure 12. Mean pressure coefficient and RMS fluctuating pressure coefficient on upper surface of deck at $-5^{\circ}$ incidence : a) Mean pressure coefficient; b) RMS fluctuating pressure coefficient. 
remaining fairly constant over the whole of the surface. Both of these results again illustrate that the region of separated flow has been suppressed, thus giving a clear indication of the strong effect the barriers have on the flow field. In the $0^{\circ}$ case, there is little difference between the barrier options in the latter half of the upper surface. There is more variation in the early part of the deck, with the full height barriers almost completely removing the suction pressures on the surface immediately behind the plates, and also producing the lowest RMS fluctuation, suggesting that this configuration has the strongest effect in removing the region of separated flow. Also, Option 2, with 4 steep plates, still has a large suction pressure at around $10 \%$ of the deck width, probably due to the high acceleration of the flow induced by the barriers as illustrated for this configuration in the velocity contour plot (Figure 8). In Option 3, there are indications of a small region of separated flow, up to approximately $30 \%$ deck width, suggesting that this option is the least affected by the inclusion of barriers, although the separated region is still much smaller than the basic deck section.

The mean and fluctuating pressures at angles of incidence further highlight the strong effect that the barriers have on the flow field around the deck. At $+5^{\circ}$ incidence (Figure 11 ), the region of separated flow on the upper surface of the basic deck section is larger than at $0^{\circ}$, with the separation zone now extending over the whole of the surface, with large suction pressures and no region of constant pressure towards the rear part of the deck. Also, the fluctuating pressures are generally much higher, especially over the latter half of the deck. In contrast, all three cases with barriers show little difference to the results from the $0^{\circ}$ case. There is a slight shift towards higher suction pressures, probably due to a greater acceleration of the flow around the upper surface of the deck at positive incidence. However, the general shape of the pressure distribution is largely unchanged, with no indication that large regions of separated flow are present. This is especially the case in Options 1 and 2, although for design Option 3, there is some evidence that the small separation region mentioned earlier has increased slightly in size, now extending up to approximately $45 \%$ deck width. The RMS pressure fluctuations are relatively unchanged from the $0^{\circ}$ case, both in terms of the magnitude and the shape of the distribution.

At $-5^{\circ}$ incidence (Figure 12), the basic deck section shows a smaller separation region, with the flow reattaching at around $40 \%$ deck width, indicated by the region of relatively constant mean pressure coefficient in the rear part of the deck. The smaller separation zone is also characterised by generally lower RMS pressure fluctuations, associated with wind predominantly along the deck surface suppressing the separation and thus reducing flow unsteadiness. Again, the cases with barriers illustrate that the pressure coefficients are relatively unchanged compared to the $0^{\circ}$ case, with similar distributions indicating almost no region of separated flow, although the slight increase in the pressure is due to the flow tending to stagnate on the upper surface of the deck at negative incidence. Options 1 and 2 show very little change in the distribution of mean and fluctuating pressure compared to $0^{\circ}$ incidence, indicating that the barriers have the strongest effect in these cases. Option 3 shows that the small separation zone has 
reduced at $-5^{\circ}$ incidence, with the flow now reattaching at approximately $20-25 \%$ deck width, again suggesting that the effect of the barriers is moderated somewhat in this case.

\section{DYNAMIC ANALYSIS}

\subsection{Flutter analysis of proposed design options}

For the dynamics analysis, a series of calculations of the section undergoing forced sinusoidal oscillations were performed using DIVEX. The simulations involved separate vertical and torsional motion about the axis at mid-chord of the section at a range of reduced velocities from $\mathrm{U}_{\mathrm{r}}=3.0$ - 12.0. The amplitudes of the motion used in the analysis were $0.05 \mathrm{~B}$ and $4^{\circ}$ for the vertical and torsional cases respectively. The method of extracting the flutter derivatives follows that outlined in Dyrbye et al (1996). The Fourier transforms of the unsteady lift and moment coefficient time histories may be represented by

$$
\begin{aligned}
& \Phi_{L}(\omega)=F_{L h}(\omega) \Xi(\omega)+F_{L \alpha}(\omega) \Lambda(\omega) \\
& \Phi_{M}(\omega)=F_{M h}(\omega) \Xi(\omega)+F_{M \alpha}(\omega) \Lambda(\omega)
\end{aligned}
$$

where $\Xi(\omega)$ and $\Lambda(\omega)$ are the Fourier transforms of the vertical and torsional deck displacements respectively. $F_{L h}$ and $F_{L \alpha}$ represent the components of the lift coefficient due to the transverse and torsional motion, and similarly, $F_{M h}$ and $F_{M \alpha}$ represent the components of the moment coefficient due to the transverse and torsional motion. For harmonic motion, these components can be represented in terms of the flutter derivatives, (21).

$$
\begin{aligned}
& F_{L h}(\omega)=\frac{1}{2} \omega^{2} \rho B^{2}\left(H_{4}^{*}(\omega)+i H_{1}^{*}(\omega)\right) \\
& F_{L \alpha}(\omega)=\frac{1}{2} \omega^{2} \rho B^{3}\left(H_{3}^{*}(\omega)+i H_{2}^{*}(\omega)\right) \\
& F_{M h}(\omega)=\frac{1}{2} \omega^{2} \rho B^{3}\left(A_{4}^{*}(\omega)+i A_{1}^{*}(\omega)\right) \\
& F_{M \alpha}(\omega)=\frac{1}{2} \omega^{2} \rho B^{4}\left(A_{3}^{*}(\omega)+i A_{2}^{*}(\omega)\right)
\end{aligned}
$$

As the DIVEX calculations were all performed using a forced sinusoidal motion in either the vertical or torsional degree of freedom, this results in one of the Fourier transforms of the body motion in Eq. 11 being zero, and so the flutter derivatives can easily be determined directly from Eq. 11 and Eq. 12 .

As determined in the static analysis, the effect of the barriers on the aeroelastic stability of the bridge was to limit the variation of aerodynamic force coefficients with incidence, particularly the moment coefficient. A low moment slope can be an indication of susceptibility of the structure to a single degree of freedom flutter instability in the torsional direction. Initially, for the basic deck and Option 1, a full set of flutter derivatives were determined and a full 2DOF coupled flutter was considered. Once the results confirmed that the cases with barriers were indeed a 1DOF instability, for the remaining deck options, a simplified 1DOF analysis in the torsional direction was used. 
For a 1DOF torsional flutter instability, a critical value of the "aerodynamic damping" can be ascertained from the equation of motion in the torsional degree of freedom. The critical condition is when the "system damping" (combined structural and aerodynamic damping) is zero. By equating the damping terms (terms involving $\dot{\alpha}$ ), and by assuming that the unknown reduced flutter frequency, $k$, is equal to the fundamental torsional frequency, $k_{\alpha}$, a critical value of the flutter coefficient, $A_{2}{ }^{*}$ crit , can be determined, Eq. (13).

$$
A_{2}{ }^{*}{ }_{c r i t}=\frac{2 I_{\alpha} \zeta_{\alpha}}{\rho B^{4}}
$$

Using the structural properties given in Table 1 , a critical value of $A_{2}{ }^{*}$ crit $=0.0459$ for the onset of 1DOF torsional flutter can be derived. For values of $A_{2}{ }^{*}$ above this critical value, the bridge will be unstable, and the critical wind speed can be obtained from the reduced velocity, $U_{r}$, Eq. (14), at which $A_{2}{ }^{*}$ is equal to the critical value.

$$
U_{r}=\frac{U_{\infty}}{f B} \quad\left(=\frac{2 \pi}{k}\right)
$$

For each deck option only the results for the flutter derivative $A_{2}{ }^{*}$ are presented (Figure 13), with the critical reduced velocities and wind speeds for each deck option shown in Table 4 . The wind speeds are all normalised with respect to the design wind speed for the proposed bridge. It should be noted that the lowest reduced velocity used in the DIVEX analysis was 3.0, and critical speeds lower than this were determined by using a spline curve fit to the data.

For the basic deck section, the $A_{2}{ }^{*}$ derivative did not exceed the critical value, although it is close to becoming unstable at a reduced velocity of approximately 5 , which would correspond to a normalised critical speed 1.53. From further analysis of the full set of flutter derivatives $\left(H_{i}\right.$ and $\left.A_{i}, i=1-4\right)$ for the basic deck section, a coupled 2 degree of freedom flutter instability was found to occur at a reduced velocity of 9.64 , with a normalised critical speed of 2.69, well in excess of the design speed. One of the initial reasons for undertaking the stability analysis of this bridge was the longer than usual span for this type of footbridge. However, this result illustrates that the basic deck exhibits a flutter instability at a wind speed significantly greater than the design speed.

In each of the deck options with barriers, $A_{2}{ }^{*}$ is either positive or close to zero, and the critical value is exceeded in the range of reduced velocities considered, indicating a greater susceptibility to single DOF torsional flutter when barriers are included on the deck.

The initial favoured design option under consideration for the bridge prior to this analysis was Option 1, with full height barriers. The results of the static analysis indicated that this configuration seems to have the strongest effect on the flow field over the upper surface of the bridge deck, with very little variation in the flow at both positive and negative angles of incidence. This is confirmed in the flutter analysis, with this configuration being found to be the most susceptible to a 1DOF torsional instability, initiated at a critical speed as low as $38.1 \%$ of the design wind speed. As 
shown in Figure 13, the flutter derivative $A_{2}{ }^{*}$ returns to the stable region at a reduced velocity just over 3.0 , corresponding to a normalised critical speed of approximately 1.0 .

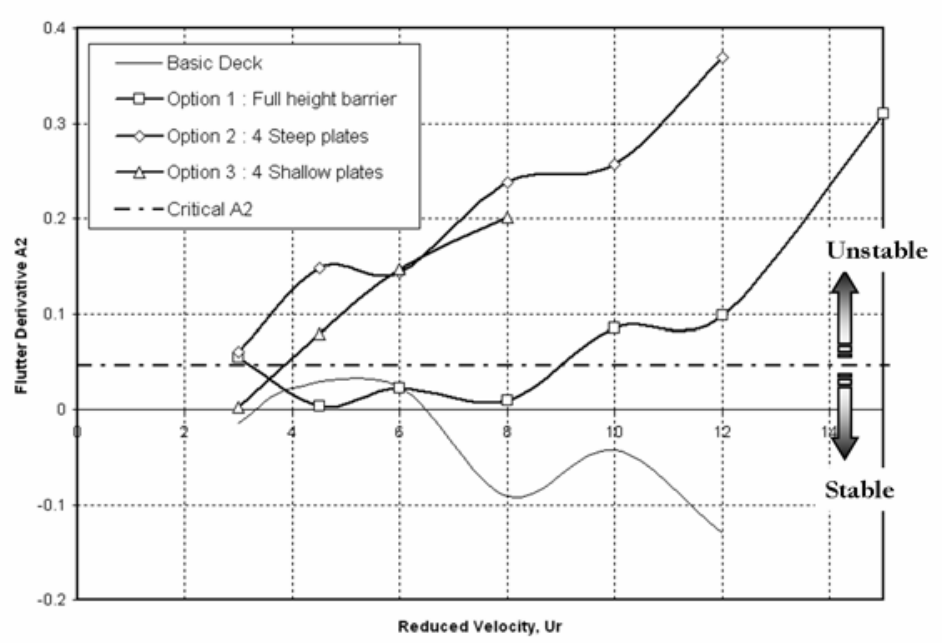

Figure 13. $A_{2}{ }^{*}$ "Torsional Aerodynamic Damping" Flutter Derivatives for each Deck Option Comparison with Critical Value.

Table 4.

Critical speeds of single DOF flutter instability for Each Bridge Option.

\begin{tabular}{lcc}
\hline Configuration & $\begin{array}{c}\text { Reduced velocity for } \\
A_{2, \text { crit }}{ }^{*}\end{array}$ & $\begin{array}{c}\text { Critical flutter speed } \\
{ }^{\dagger}\end{array}$ \\
\hline Option $1:$ Full height flat plates. & 1.25 & 0.381 \\
Option $2: 4$ steep flat plates. & 2.74 & 0.838 \\
Option $3: 4$ shallow flat plates. & 3.87 & 1.186 \\
$\begin{array}{l}\text { Option } 3: \text { Using structural properties of } \\
\text { Deck A. }\end{array}$ & 4.088 & 2.733 \\
$\begin{array}{l}\text { Deck A } \\
\begin{array}{l}\text { Deck A : Using structural properties of } \\
\text { proposed bridge. }\end{array}\end{array}$ & 4.682 & 3.176 \\
\hline
\end{tabular}

${ }^{\dagger}$ Critical flutter speed normalised with respect to design wind speed, $U_{\text {crit }} / U_{\text {des }}$.

The unstable nature of the initial design option led to necessary redesign of the barriers to improve the structural integrity of the bridge. Option 2 was the first redesign, obtained simply by removing most of the upper flat plates from the barriers to reduce the turning effect on the flow, while maintaining the steep angle of the plates to retain one of the key architectural elements of the design. From the static analysis, it was found that, despite reducing the number of flat plates in the barrier, the flow over the upper deck was still very much determined by the barriers, even at angles of incidence. 
In terms of the stability of the deck, a 1DOF torsional flutter instability was found at a critical speed of $83.8 \%$ of the design speed. The susceptibility of the bridge to flutter is still strongly dominated by the effect of the barriers on the flow, although reducing the number of flat plates has improved the stability of the bridge, with a significant increase in the critical speed. However, the flutter instability is still well below the design speed, and so a further redesign of the barrier was required.

Option 3 kept the reduced number of flat plates, but significantly reduced their angle to the deck, to further moderate the turning effect of the barriers at the edges of the deck. The angle was reduced to what the designers considered to be close the minimum value that could be used whilst still restricting the view of horses crossing the bridge. From the static analysis, the barriers in this configuration were found to still have a strong effect on the flow, but not to the same extent as the design options 1 and 2, as discussed in the static analysis. This modification successfully increased the flutter speed to a value above the design speed. Although the critical speed is significantly lower than for the basic deck, the 1DOF torsional flutter instability now occurs at a speed $18.6 \%$ higher than the design wind speed. Initially, it was judged that this critical speed may be a little too close to the design speed, and that further modification, or even a complete change in barrier design may be required to achieve the necessary structural integrity. However, on further investigation, it was determined that the calculated design speed for this location already included a "safety margin". Hence, the predicted critical wind speed for 1DOF torsional flutter was considered as incorporating a sufficient overall margin of safety, and therefore deck Option 3 was selected as the barrier design.

\subsection{Validation of flutter analysis.}

Extensive validation of DIVEX for determining aerodynamic characteristics and flutter derivatives for bluff bodies has been previously undertaken, full details of which are given elsewhere (Taylor and Vezza, 2001, 2002a; Taylor et al 2002b). For example, flutter derivatives on streamline and bluff deck sections have been successfully predicted and critical flutter speeds have been accurately determined to within a few $\mathrm{m} / \mathrm{s}$. Also, DIVEX has been used previously in design studies for bridge deck flutter, providing accurate results for both static and dynamic aerodynamic characteristics, sometimes in advance of experimental details being available. These results give confidence in the numerical method, to give a reliable prediction on a new geometry where no previous information or experimental data is available.

The investigation of this new bridge, by definition, is an extension to previous work and hence there is limitation on the particular validation of this configuration. However, structural properties and wind tunnel results of a previous bridge that incorporated a similar barrier configuration were made available by Halcrow, to allow further investigation on how DIVEX performs on this type of configuration. Due to commercial confidentiality this alternative bridge is simply labelled "Deck A". Importantly, this bridge had not experienced the sensitivity to the barriers and had not demonstrated a strong flutter instability either in wind tunnel tests or in service. 
A brief numerical investigation into Deck A was performed using DIVEX, in an attempt to understand these results, and also to assess why Deck A appears to be so much more stable, with the results summarised in Table 4 and Figure 14. From the DIVEX predictions of the flutter derivatives for Deck A, a non-dimensional critical flutter speed of 3.176 was predicted. Wind tunnel tests indicated that Deck A would be stable up to a non-dimensional speed of around 1.7, with no critical speed being determined. The DIVEX results are therefore qualitatively in agreement with the wind tunnel results, that Deck A appears to be a much more stable structure.

It was ascertained that two main factors contribute to the greater stability of Deck A. Firstly, Deck A has very different structural characteristics to the proposed new bridge, being slightly heavier, significantly stiffer, with higher fundamental frequencies, a greater torsional to bending frequency ratio and a significantly wider deck section. These differences reduce the susceptibility of the structure to flutter instabilities by increasing the critical value of $A_{2} *$ at which there is zero system damping. The effect of the structural properties is confirmed when the flutter derivatives for Deck A are used along with the structural properties for the proposed bridge. The non-dimensional speed for 1DOF torsional flutter is reduced to 1.338 (Table 4). Similarly, if the structural properties of Deck A are applied to the new bridge, the flutter speed is raised from 1.186 to 2.773 for Option 3.

The second factor contributing to the greater stability of the alternative deck the different arrangement of the leading edge. For the new bridge, the deck is essentially a flat plate between two rectangular sections, whereas Deck A is a flat plate between two circular sections (Figure 14). The different arrangements at the leading edge have a significant effect on the flow around the basic deck sections, and hence on the flow approaching the barriers. For Deck A, the rounded leading edge significantly reduces the angle at which the shear layer separates from the leading edge, and the size of the separated flow region on the upper surface is reduced. Hence, the flow over Deck A already has a much larger horizontal component, so that when the barriers are included, their turning effect has less impact on the flow field over the upper deck surface.

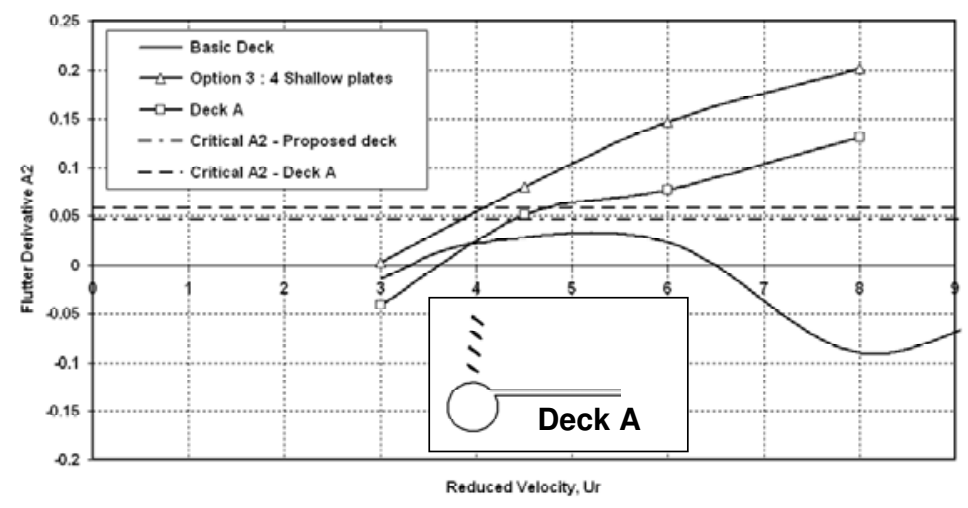

Figure 14. Comparison of $A_{2}{ }^{*}$ Flutter Derivative and critical values for proposed deck section and alternative Deck A. 
Although this does not provide a full validation of DIVEX for this configuration, the qualitative agreement of the results with the experimental information from the previous deck provides some additional measure of confidence in the numerical approach.

\section{CONCLUSIONS}

A numerical study into the aerodynamic characteristics and aeroelastic stability of a proposed footbridge has been undertaken, with the main conclusions being :

- The vortex method, DIVEX, has been demonstrated as a useful and effective design tool for assessing candidate bridge deck configurations, allowing various design options to be considered.

- The inclusion of angled flat plates at the edges of the deck section has a strong effect on the aerodynamic characteristics of the deck.

- Flow visualisation demonstrates the flat plates acting strongly as flow guide vanes, directing the flow downwards at the leading edge and upwards at the trailing edge.

- The flat plates have a strong effect on the static aerodynamic loads of the sections lower lift, higher drag and moment at $0^{\circ}$, as well as an order of magnitude reduction in moment slope.

- The basic deck section, with no flat plates, experiences a two degree of freedom flutter instability, with a critical flutter speed well in excess of the design speed.

- Including the pedestrian barriers significantly increases the susceptibility of the footbridge to a flutter instability.

- Critical flutter speed of the deck sections are strongly affected by the configuration and angle of flat plates. Deck options 1 and 2, both with steeply angled flat plates have critical speeds, or are unstable below the design wind speed.

- Deck option 3, with a small number of shallow angled flat plates is stable with respect to flutter up to a wind speed approximately $18.6 \%$ greater than the design wind speed. In conjunction with a built-in safety margin, this was considered to be sufficiently far from the design speed to be a viable design option.

- An alternative deck section based on a previous design was considered, and was found to have a critical flutter speed approximately $33.8 \%$ greater than the design wind speed.

\section{REFERENCES}

Chen, X., Kareem, A., 2003. Anatomy of Bridge Flutter : Some New Insights. Proceedings of 11th International Conference on Wind Engineering, Lubbock, Texas, USA, 2-5 June 2003.

Dallard, P., Fitzpatrick, A.J., Flint, A., Le Bourva, S., Low, A., Ridsdill Smith, R.M., Willford, M., 2001. The London Millennium Footbridge. Structural Engineer 79(22), 17-33.

Dyrbye, C., Hansen, S.O., 1996. Wind Loads on Structures. John Wiley and Sons. (English edition.) Flaga, A., Bosak, G., Michalowski, T., 2002. Study of aerodynamical behaviour of suspension footbridges against wind load. International Journal of Fluid Mechanics Research 29(3-4), 363-376. Larsen, A., Walther, J.H., 1997. Aeroelastic Analysis of Bridge Girder Sections Based on Discrete Vortex Simulations. Journal of Wind Engineering and Industrial Aerodynamics 67-68, 253-265.

Leonard, A., 1980. Vortex Methods for Flow Simulation. Journal of Computational Physics 37, $289-335$. 
Lin, H., 1997. Prediction of Separated Flows Around Pitching Aerofoils using a Discrete Vortex Method. Ph.D. Thesis 10896, Department of Aerospace Engineering, University of Glasgow, Scotland, UK Mackenzie, D.K., Morgenthal, G., McRobie, F.A., 2002. Wind shielding of the M4 at Neath. Proceedings of 5th UK Conference on Wind Engineering, Nottingham, U.K., 4-6 September 2002. Matsumoto, M., Kobayashi, Y., Shirato, H., 1996. The Influence of Aerodynamic Derivatives on Flutter. Journal of Wind Engineering and Industrial Aerodynamics 60(1-3), 227-239

Pirner, M., Fischer, O., 1998. Wind-induced vibrations of concrete stress-ribbon footbridges. Journal of Wind Engineering and Industrial Aerodynamics 74-76, 871-881.

Puckett, E.G., 1993. Vortex Methods : An Introduction and Survey of Selected Research Topics. Incompressible Computational Fluid Dynamics, ed. M.D. Gunzb and R.A. Nicolaides, Cambridge University Press.

Sarpkaya, T., 1989. Computational Methods with Vortices - The 1988 Freeman Scholar Lecture. Journal of Fluids Engineering 111, 5-52.

Scanlan, R.H., Tomko, J.J., 1971. Airfoil and Bridge Deck Flutter Derivatives. Journal of the Engineering Mechanics Division 97, 1717-1737.

Scanlan, R.H., 1978. The Action of Flexible Bridges Under Wind, I : Flutter Theory. Journal of Sound and Vibration 60(2), 187-199.

Scanlan, R.H., 1997. Some Observations on the State of Bluff-Body Aeroelasticity. Journal of Wind Engineering and Industrial Aerodynamics 69-71, 77-90.

Simiu, E., Scanlan, R.H., 1996. Wind Effects on Structures : Fundamentals and Applications to Design. 3rd Edition, John Wiley and Sons.

Tanaka, T., Yoshimura, T., Gimsing, N.J., Mizuta, Y., Kang, W.-H., Sudo, M., Shinohara, T., Harada, T., 2002. A study on improving the design of hybrid stress-ribbon bridges and their aerodynamic stability. Journal of Wind Engineering and Industrial Aerodynamics 90(12-15), 1995-2006.

Taylor, I.J., Vezza, M., 1999a. Prediction of unsteady flow around square and rectangular section cylinders using a discrete vortex method. Journal of Wind Engineering and Industrial Aerodynamics 82(1-3), 247-269.

Taylor, I.J., Vezza, M., 1999b. Calculation of the Flow Field around a Square Section Cylinder undergoing Forced Transverse Oscillations using a Discrete Vortex Method. Journal of Wind Engineering and Industrial Aerodynamics 82(1-3), 271-291.

Taylor, I.J., Vezza, M., 2001. Application of a discrete vortex method for the analysis of suspension bridge deck section. Wind and Structures 4(4), 333-352.

Taylor, I.J., Vezza, M., 2002a. Aeroelastic stability analysis of a bridge deck with added vanes using a discrete vortex method. Wind and Structures 5(2-4), 277-290.

Taylor, I.J., Vezza, M., Withycombe, S.I., 2002b. Commercial computational wind engineering :

Contributions of DIVEX to recent design projects. Proceedings of 5th UK Conference on Wind

Engineering, Nottingham, U.K., 4-6 September 2002.

The Highways Agency, 2001. Design Rules for Aerodynamic Effects on Bridges. BD 49/01, Vol. 1, Sect. 3, Part 3

Vejrum, T., Queen, D.J., Larose, G.L., Larsen, A., 2000. Further aerodynamic studies of Lions' Gate Bridge - 3 lane renovation. Journal of Wind Engineering and Industrial Aerodynamics 88(2-3), 325-341. 